\title{
Complex Spin Texture in the Pure and Mn-Doped Topological Insulator $\mathrm{Bi}_{2} \mathrm{Te}_{3}$
}

\author{
J. Henk, ${ }^{1, *}$ A. Ernst, ${ }^{1}$ S. V. Eremeev, ${ }^{2,3,4}$ E. V. Chulkov, ${ }^{4,5,6}$ I. V. Maznichenko, ${ }^{7}$ and I. Mertig ${ }^{1,7}$ \\ ${ }^{1}$ Max-Planck-Institut für Mikrostrukturphysik, Weinberg 2, D-06120, Halle, Germany ${ }^{\dagger}$ \\ ${ }^{2}$ Institute of Strength Physics and Materials Science, pr. Academicheskii 2/4, 634021, Tomsk, Russia \\ ${ }^{3}$ Tomsk State University, pr. Lenina 36, 634050, Tomsk, Russia \\ ${ }^{4}$ Donostia International Physics Center (DIPC), Paseo de Manuel Lardizabal, 4, 20018 San Sebastián/Donostia, \\ Basque Country, Spain \\ ${ }^{5}$ Departamento de Física de Materiales, Facultad de Ciencias Químicas, UPV/EHU, Apartado 1072, 20080 San Sebastián, \\ Basque Country, Spain \\ ${ }^{6}$ Centro de Física de Materiales, CFM-MPC, Centro Mixto CSIC-UPV/EHU, Apdo. 1072, 20080 San Sebastián/Donostia, \\ Basque Country, Spain \\ ${ }^{7}$ Institut für Physik, Universität Halle-Wittenberg, Von-Seckendorff-Platz 1, D-06120 Halle, Germany
}

(Received 24 October 2011; published 14 May 2012)

Topological insulators are characterized by the presence of spin-momentum-locked surface states with Dirac points that span the fundamental bulk band gap. We show by first-principles calculations that the surface state of the insulator $\mathrm{Bi}_{2} \mathrm{Te}_{3}$ survives upon moderate $\mathrm{Mn}$ doping of the surface layers. The spin texture of both undoped and $\mathrm{Mn}$-doped $\mathrm{Bi}_{2} \mathrm{Te}_{3}$ is much more complicated than commonly believed, showing layer-dependent spin reversal and spin vortices.

DOI: 10.1103/PhysRevLett.108.206801

PACS numbers: 73.20.At, 71.70.Ej, 75.70.Rf

Topological insulators are a new class of materials with promising properties for both fundamental physics and technological applications for upcoming spin-electronic devices [1,2]. The topological character in threedimensional topological insulators, like $\mathrm{Bi}_{2} \mathrm{Se}_{3}$ and $\mathrm{Bi}_{2} \mathrm{Te}_{3}$, manifests itself by a conducting, linearly dispersed Dirac surface state bridging the bulk band gap. A striking feature of topological insulators is that their surface states are topologically protected: They are robust against perturbations that maintain time-reversal symmetry [3]. However, if time-reversal symmetry is broken due to magnetic order the topological character can be strongly modified $[1,4]$.

A Dirac state shows a unique spin texture of the twodimensional electron gas which can cause, e.g., the spinHall effect [5-7]. In the Rashba-Bychkov effect, the spin degeneracy of the electronic states is lifted but their dispersion curvature is maintained [8,9]. In contrast, the surface electronic structure in topological insulators [10,11] can be viewed as if the two branches have opposite dispersion curvature and touch at a single point, i.e., at the Dirac point. This feature gives rise to the quantum spin-Hall effect $[12,13]$ or to a magnetic monopole [14].

In this Letter, we provide strong support by a theoretical first-principles investigation that the surface state of the topological insulator $\mathrm{Bi}_{2} \mathrm{Te}_{3}$ that is doped with $\mathrm{Mn}$ in the topmost quintuple layer "survives" but with modified dispersion relation and spin texture. But more strikingly, the spin textures in both $\mathrm{Bi}_{2} \mathrm{Te}_{3}$ and $\mathrm{Bi}_{2-x} \mathrm{Mn}_{x} \mathrm{Te}_{3}$ deviate significantly from those commonly believed. More precisely, they exhibit spin reversal in the topmost Ti layer and spin vortices.

$\mathrm{Bi}_{2} \mathrm{Te}_{3}$ is assembled by so-called quintuple layers (QLs) that are weakly van der Waals bound and are stacked along the crystallographic (111) axis. The quintuple layers comprise five strongly bound hexagonal layers which contain two atomic Bi layers separated by atomic Te layers (Fig. 1). Because of the complicated geometric structure, the spin-resolved electronic structure is feature rich [15] and, as we will show, differs significantly from that obtained by model calculations (cf. [16]).

Hsieh et al showed experimentally that $\mathrm{Bi}_{2} \mathrm{Te}_{3}$ can be moderately doped with Mn [17]. In STM experiments, Hor et al found a substitutional surface alloy in which Bi atoms are randomly replaced by $\mathrm{Mn}$ atoms [18]. Thus in our first-principles calculations, we replace each $\mathrm{Bi}$ atom by $\mathrm{Bi}_{0.9} \mathrm{Mn}_{0.1}$ in the topmost QL (Mn concentration $10 \%$ ),

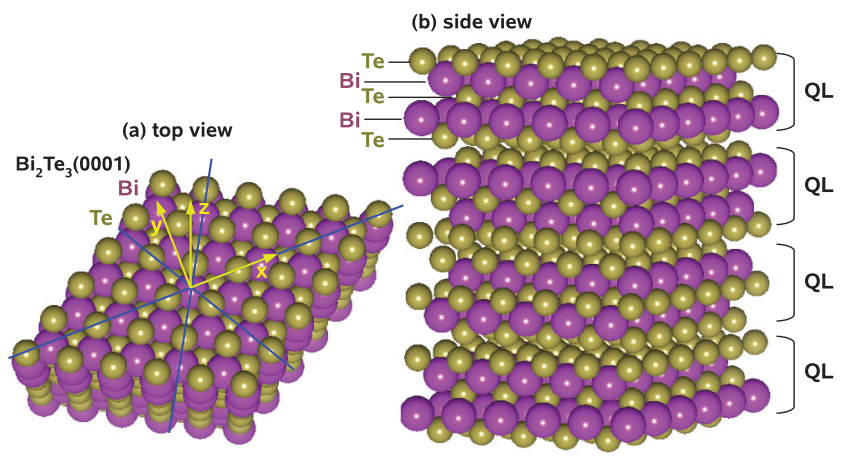

FIG. 1 (color). (111) surface of $\mathrm{Bi}_{2} \mathrm{Te}_{3}$. (a) Top view. $\mathrm{Bi}$ (magenta) and Te (brown) atoms form hexagonal layers with threefold rotational symmetry. The blue lines highlight mirror axes of the surface ( $x$ and $y$ axis in-plane, $z$ axis out-of-plane). (b) Side view. A stack of four quintuple layers (QLs, indicated on the right) is shown, each consisting of an alternating stacking of two $\mathrm{Bi}$ and three Te layers. 
treated as an inhomogeneous binary substitutional alloy $\mathrm{Bi}_{2-x} \mathrm{Mn}_{x} \mathrm{Te}_{3}$.

First-principles calculations were performed within the local spin-density approximation to density functional theory, with Perdew-Wang parametrization for the exchangecorrelation functional [19]. The electronic and magnetic properties were obtained by a multiple-scattering theoretical approach (scalar-relativistic and relativistic KorringaKohn-Rostoker methods [20-22]).

Geometrical relaxations at the surface can be safely neglected, as has been checked by total-energy calculations. Mn-doped samples were taken as substitutional binary alloys in which the Bi sites in the topmost QL are replaced by $\mathrm{Bi}_{0.9} \mathrm{Mn}_{0.1}$ sites, in agreement with STM experiments [18]. The disorder is treated within the coherent potential approximation [23].

Detailed information on the spin-resolved electronic structure is obtained from the Bloch spectral density

$$
N_{i \alpha}\left(E, \vec{k}_{\|}\right)=-\left.\frac{1}{\pi} \operatorname{Im} \operatorname{Tr} G_{i i}^{+}\left(E, \vec{k}_{\|}\right)\right|_{\alpha}
$$

where $G_{i i}^{+}\left(E, \vec{k}_{\|}\right)$is the site- and momentum-resolved Green function of the system at energy $E\left[i\right.$ site index, $\vec{k}_{\|}=$ $\left(k_{x}, k_{y}\right)$ wave vector parallel to the layers]. By restricting the trace to a subset $\alpha$ of quantum numbers, we obtain, e.g., the orbital composition and spin difference of the electronic state under consideration. The spin structure is represented as spin projections

$$
S_{i \mu}\left(E, \vec{k}_{\|}\right) \equiv N_{i \mu \uparrow}\left(E, \vec{k}_{\|}\right)-N_{i \mu \downarrow}\left(E, \vec{k}_{\|}\right),
$$

with the spin quantization axis $\mu=x, y$, or $z$.

From $a b$ initio computed Heisenberg exchange constants, we deduce a ferromagnetically ordered state with Curie temperature of about $12 \mathrm{~K}$ (experimental result $9-12 \mathrm{~K}$ for a $\mathrm{Mn}$ concentration of $9 \%$ [18]). The local magnetic moments are $4.58 \mu_{B}$ (second layer) and $4.52 \mu_{B}$ (fourth layer); Niu et al. find $4.0 \mu_{B}$ for bulk $\mathrm{Bi}_{2-x} \mathrm{Mn}_{x} \mathrm{Te}_{3}$ [24].

Since the magnetization direction $\vec{M}$ can be controlled by an external magnetic field, we investigated the electronic structure for $\vec{M} \| \vec{x}$ [in-plane, within the $x z$ mirror

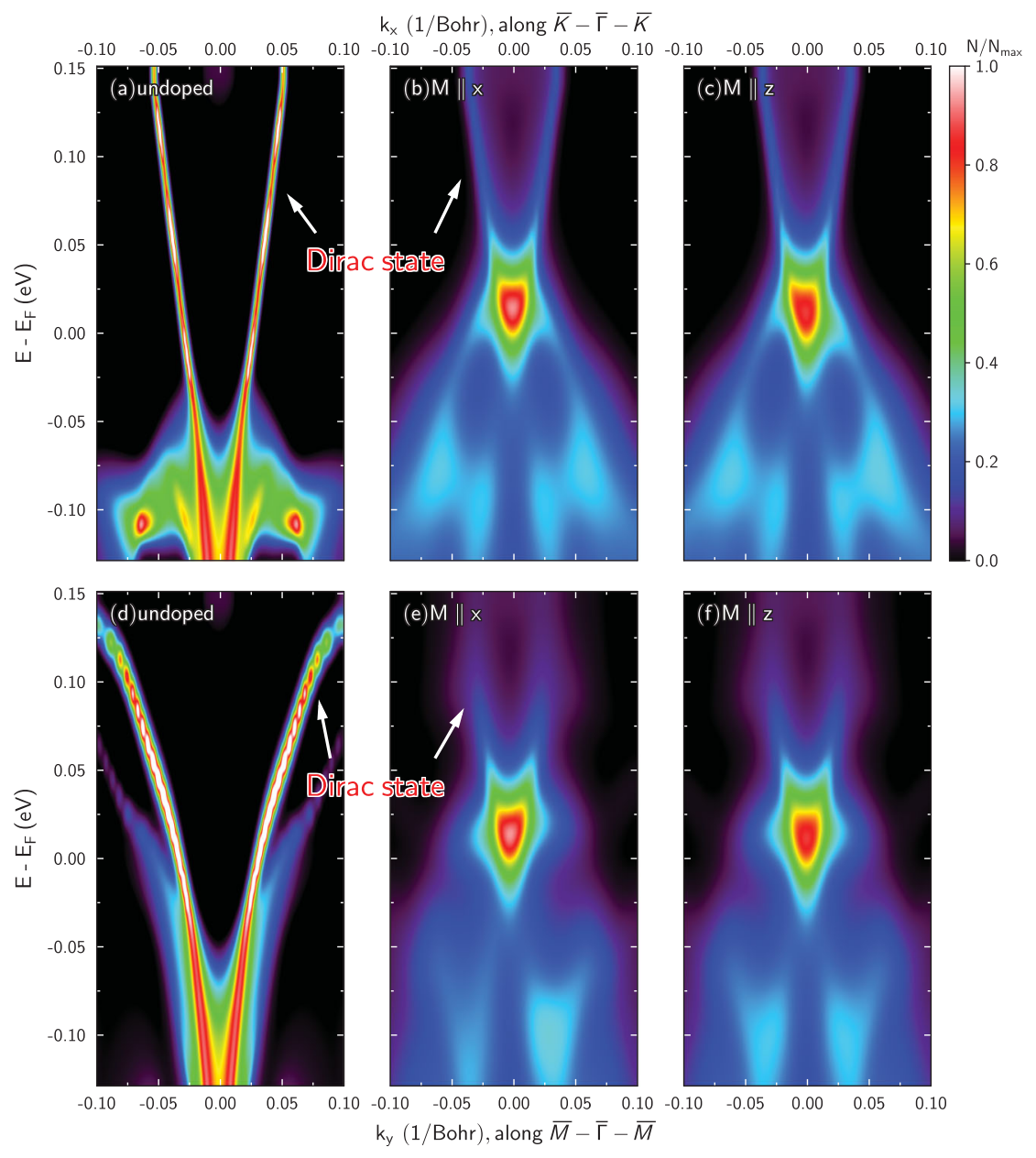

FIG. 2 (color). Dispersions $\left(E, \vec{k}_{\|}\right)$of the Dirac state in $\mathrm{Bi}_{2} \mathrm{Te}_{3}$ [undoped; (a) and (d)] and $\mathrm{Bi}_{2-x} \mathrm{Mn}_{x} \mathrm{Te}_{3}$ (doped; $10 \% \mathrm{Mn}$ with magnetization $\vec{M}$ parallel to $x$ (b), (e) and $z$ (c), (f)] for electron momenta $\vec{k}=\left(k_{x}, k_{y}\right)$ along $\vec{k}_{x}$ (i.e., $\bar{K}$ - $\bar{\Gamma}-\bar{K}$ in the two-dimensional Brillouin zone; top row) and $\vec{k}_{y}$ (i.e., $\bar{M}-\bar{\Gamma}-\bar{M}$; bottom row). The linear color scale presents the spectral weight of a top-layer Te site, normalized in each panel. The Fermi level is at $0 \mathrm{eV}$. 
plane of the (111) surface], $\vec{M} \| \vec{y}$ (in-plane, normal the mirror plane), and $\vec{M} \| \vec{z}$ (normal to the surface; out-ofplane magnetic anisotropy was found experimentally by Hor et al [18]). Because the dispersion relations for $\vec{M} \| \vec{x}$ and $\vec{M} \| \vec{y}$ are very similar we do not show data for $\vec{M} \| \vec{y}$.

The Dirac surface state at the (111) surfaces shows up as a single band of conical shapes crossing the fundamental bulk band gap [25]. Constant-energy cuts through the dispersion relation $E\left(\vec{k}_{\|}\right)$are circular at energies close to the Dirac point. The surface state's spin $\vec{s}$ is perpendicular to the surface-parallel component of the electron momentum $\hbar \vec{k}_{\|}$, as is typical for electronic states influenced by Rashba-Bychkov spin-orbit coupling [26,27]. At larger energy distances from the Dirac point, these momentum distributions become hexagonally shaped and eventually warped. This warping is accompanied by an increasing out-of-plane spin component $s_{z}$ [28,29]. Note that the heavy element $\mathrm{Bi}$ induces strong spin-orbit coupling effects in $\mathrm{Bi}_{2} \mathrm{Te}_{3}$ [30], similar to the surface alloy $\mathrm{Bi} / \operatorname{Ag}(111)[8,9]$.

Our calculations show that the Dirac state of undoped $\mathrm{Bi}_{2} \mathrm{Te}_{3}$ disperses linearly along $\vec{k}_{x}[\bar{K}-\bar{\Gamma}-\bar{K}$, Fig. 2(a)] but displays a "curved" dispersion along $\vec{k}_{y}[\bar{M}-\bar{\Gamma}-\bar{M}$, Fig. 2(d)], which clearly indicates warping (cf. Refs. [24,31]). The blurred blue and green regions mainly below the Fermi energy $E_{F}$ in Fig. 2 are attributed to the surface part of bulk electronic states.

A first important result for $\mathrm{Bi}_{2-x} \mathrm{Mn}_{x} \mathrm{Te}_{3}$ is that the surface state of $\mathrm{Bi}_{2} \mathrm{Te}_{3}$ "survives" Mn doping in the topmost QL. It shows linear dispersion, in particular, along $\vec{k}_{x}$
[Figs. 2(b) and 2(c); cf. Niu et al [24]) and crosses the fundamental band gap. A second important feature is that the "curved" dispersion along $\vec{k}_{y}$ [Figs. 2(e) and 2(f)] is changed to a linear one whose density, however, becomes weak and blurred with increasing energy, due to disorder and hybridization with bulk electronic states. Further, the surface state's spectral density in $\mathrm{Bi}_{2-x} \mathrm{Mn}_{x} \mathrm{Te}_{3}$ has a clear maximum that shows up as a red spot in Figs. 2(b)-2(f), in contrast to $\mathrm{Bi}_{2} \mathrm{Te}_{3}$. Because the data in Fig. 2 are normalized to the maximum in each panel, the Dirac state with its weak spectral weight appears blueish for doped samples. Given the moderate $\mathrm{Mn}$ doping (Mn concentration of $10 \%$ ), it consists mainly of the same orbitals as its $\mathrm{Bi}_{2} \mathrm{Te}_{3}$ counterpart, except for the Mn orbitals. Both findings-maintaining the Dirac state with a weak weightagree nicely with experimental results from photoelectron spectroscopy for $\mathrm{Bi}_{2-x} \mathrm{Mn}_{x} \mathrm{Te}_{3}$ with $9 \% \mathrm{Mn}$ doping [18] and to some extend to $\mathrm{Bi}_{2} \mathrm{Se}_{3}$ with $\mathrm{Cs}$ and $\mathrm{Gd}$ doping [32] as well as to $\mathrm{Fe}$-doped $\mathrm{Bi}_{2} \mathrm{Te}_{3}$ [33]. Co adatoms deposited on $\mathrm{Bi}_{2} \mathrm{Se}_{3}$ were found to open a surface band gap [34].

Given the similarities and differences in the dispersion relations, one might ask whether the spin textures in $\mathrm{Bi}_{2} \mathrm{Te}_{3}$ and $\mathrm{Bi}_{2-x} \mathrm{Mn}_{x} \mathrm{Te}_{3}$ agree likewise. To answer this question, we first address the spin texture of $\mathrm{Bi}_{2} \mathrm{Te}_{3}$.

The topmost $\mathrm{QL}$ in $\mathrm{Bi}_{2} \mathrm{Te}_{3}$ shows the prescribed spin texture, namely, a clockwise rotating in-plane spin projection $\left(S_{x}, S_{y}\right)$ and an alternating out-of-plane spin projection $S_{z}$ [Figs. 3(a)-3(c)], in agreement with those obtained by a model Hamiltonian $[4,16]$ and in previous calculations (e.g., Refs. [29,35]). This spin texture also shows up in the subsurface layers [Figs. 3(d)-3(f)], with a

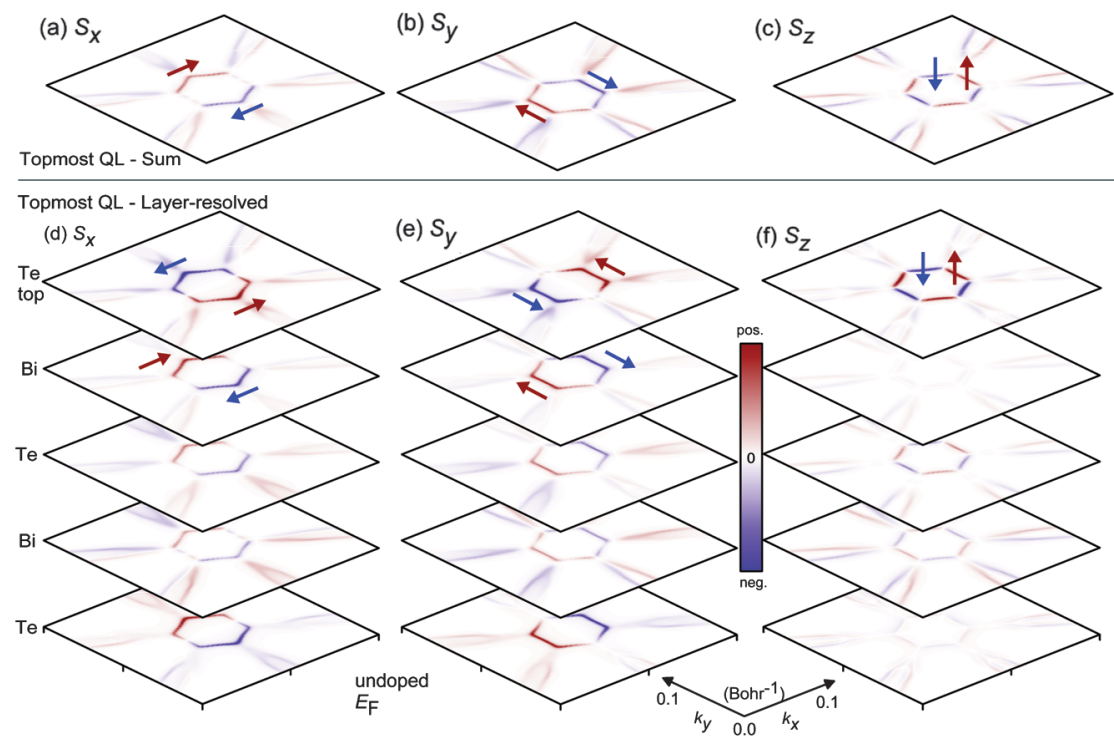

FIG. 3 (color). Spin texture of the Dirac state in the topmost QL of $\mathrm{Bi}_{2} \mathrm{Te}_{3}$, as represented by spin differences $S_{x}$, $S_{y}$, and $S_{z}$ at the Fermi energy. Spin differences are shown for the entire QL ["Topmost QL-Sum"; (a)-(c)] and resolved for each layer of the QL ["Topmost QL-Layer-resolved"; (d)-(f)]. Each square displays a central part of the two-dimensional Brillouin zone in perspective view. The color scale, with (blue, white, red) indicating (negative, zero, positive) values, is identical for all sheets and, thus, allows a visual comparison of the layerwise spin difference's strength. Blue and red arrows visualize the direction of the electron's spin projection. 
comparably weak $S_{z}$. However, the spin projection in the topmost Te site is reversed: The in-plane spin projection rotates counterclockwise. And further, the out-of-plane projection is as strong as the in-plane components, giving rise to an out-of-plane tilt angle of up to $45^{\circ}$ [36]. Further, $S_{x}$ (a) and $S_{y}$ (b) are weaker in the central layers than in the outer layers of the QL. We attribute these findings to significantly weaker localization of the topological surface state in the central atomic layers of the QL compared to that in the outer atomic layers [37]. A group-theoretical decomposition of the surface state shows that the character of $p$ states changes from dominating $p_{x \uparrow}$ in the top Te layer to $p_{y \downarrow}$ and $p_{z \downarrow}$ in all other Te layers which can cause a sign change in the potential gradient that enters the spin-orbit interaction and can reverse the spin orientation in the top layer. The spin reversal in the topmost Te layer results in a reduced spin polarization which can be measured by spin- and angle-resolved photoelectron spectroscopy [38].

Another deviation from the model spin texture shows up at larger energies at which the constant-energy contours show warping: The in-plane spin projection in the top $\mathrm{Te}$ layer [arrows in Fig. 4(a)] follows roughly an anticlockwise rotation at the "arcs" of the contour, as is consistent with Figs. 3(a) and 3(b). Exactly at the cusps, however, its rotation direction is reversed. We explain these "spin vortices" as follows. At low energies, the constant-energy contour (CEC) of the surface state is almost circular and the spin texture follows that of the Rashba model: the electron spin circulates around the Brillouin zone center, as can be regarded as a spin vortex. With increasing energy, the CEC becomes warped which results in an out-of-plane tilt of the electron spin [16] in addition to the beforementioned (in-plane) spin vortex. This scenario would hold for all energies if there were no other electronic states. However, when approaching the conduction band regime, the Dirac state hybridizes with the surface parts of bulk states in regions close to the six cusps of the heavily warped CECs. Because the surface-located parts of bulk states are also affected by the spin-orbit interaction, these states become spin-polarized at the surface [39]. The hybridization then gives rise to the six spin vortices at the cusps (We show that the Dirac state in $\mathrm{Bi}_{2} \mathrm{Te}_{3}$ hybridizes with bulk electronic states in the $\bar{\Gamma}-\bar{M}$ direction in the Supplemental Material [40] by means of its localization at the surface). In summary, there are two types of spin vortices: one solely due to the Rashba spin-orbit interaction at the Brillouin zone center, a second due to Rashba spin-orbit interaction and hybridization of the Dirac state with bulk states. We note that the above Korringa-KohnRostoker results for $\mathrm{Bi}_{2} \mathrm{Te}_{3}$ are fully confirmed by our VASP calculations but deviate in details by recent WIEN2K calculations which do not show spin reversal and vortices [29].

We now compare the spin texture in undoped $\mathrm{Bi}_{2} \mathrm{Te}_{3}$ with that of $\mathrm{Bi}_{2-x} \mathrm{Mn}_{x} \mathrm{Te}_{3}$. The in-plane projection of $\mathrm{Bi}_{2-x} \mathrm{Mn}_{x} \mathrm{Te}_{3}$ exhibits also counterclockwise rotation. Its sign, however, becomes reversed at the raylike features, similar to $\mathrm{Bi}_{2} \mathrm{Te}_{3}$ and again a clear indication of hybridization. The out-of-plane projection agrees roughly with that of $\mathrm{Bi}_{2} \mathrm{Te}_{3}$ (d)-(f). At the Brillouin zone center $\left(\vec{k}_{\|}=0\right)$, a small but finite polarization shows up for the
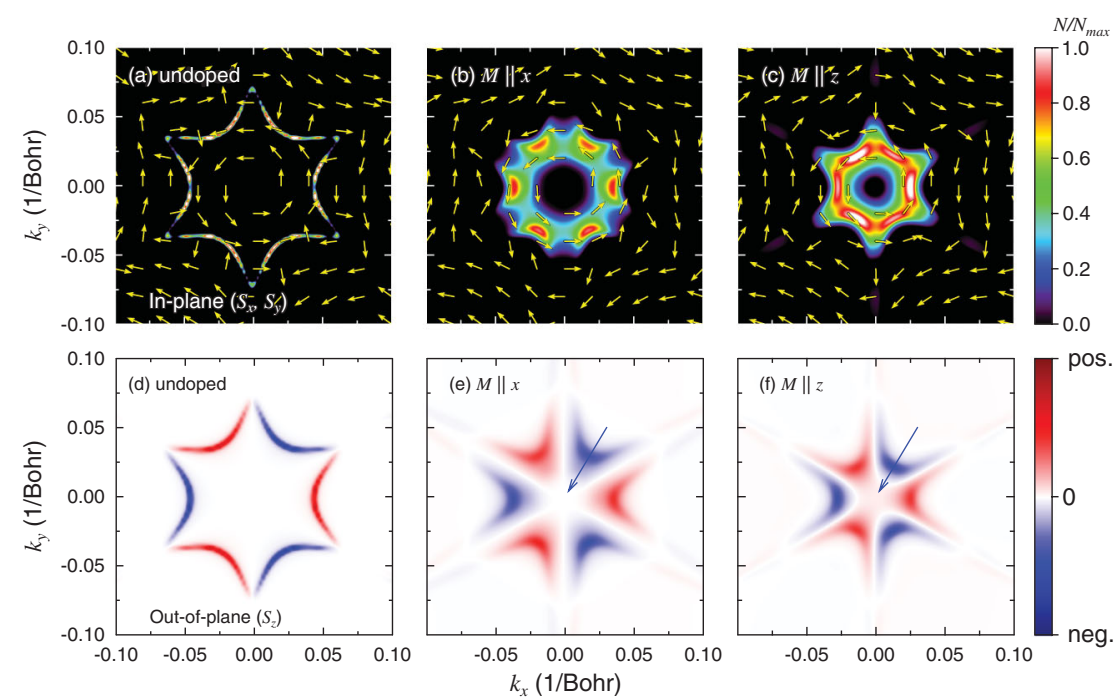

FIG. 4 (color). Spin texture of the Dirac state for $\mathrm{Bi}_{2} \mathrm{Te}_{3}$ (a), (d) and $\mathrm{Bi}_{2-x} \mathrm{Mn}_{x} \mathrm{Te}_{3}$ with magnetization $\vec{M} \| \vec{x}$ (b), (e) and $\vec{M} \| \vec{z}$ (c), (f), as represented by spin projections $S_{\mu}(\mu=x, y, z)$ in a topmost Te site. (a)-(c) In-plane spin texture. Arrows illustrate the spin orientation at the $\vec{k}_{\|}$at the arrows' tails. The color scale on the right represents the spin-integrated spectral weight $\sqrt{S_{x}^{2}+S_{y}^{2}}$ normalized in each panel, whereas arrows sketch the in-plane spin projection $\left(S_{x}, S_{y}\right)$. (d)-(f) Out-of-plane spin texture. The color scale on the right, with (blue, white, red) indicating (negative, zero, positive) values, represents the projection $S_{z}$ normalized in each panel. Arrows highlight a zero [white, in (e)] or positive [light red, in (f)] spin difference $S_{z}$ at the Brillouin zone center. The energy is $E_{F}+0.1 \mathrm{eV}$. 
sample with $\vec{M} \| \vec{z}$ [light red in (f), marked by an arrow]; this exchange splitting is missing for $\vec{M} \| \vec{x}$ [white, arrow in (e)].

The surface states in $\mathrm{Bi}_{2-x} \mathrm{Mn}_{x} \mathrm{Te}_{3}$ with magnetization along $\vec{x}$ or $\vec{z}$ show similar characteristic features as their counterpart in $\mathrm{Bi}_{2} \mathrm{Te}_{3}$. Thus, we regard them as Dirac states, although time-reversal invariance is broken by the magnetism.

In summary, our calculations give detailed insight into the spin-resolved dispersion of the surface state with and without Mn doping. Our results agree in general with spinintegrated photoemission data by Hsieh et al and Hor et al $[15,18]$, but details concerning a layerwise resolution of the spin texture call for corroboration by advanced spinresolved experiments.

*Corresponding author.

juergen.henk@physik.uni-halle.de

'Present address: Institut für Physik, Universität HalleWittenberg, Von-Seckendorff-Platz 1, D-06120 Halle, Germany

[1] Y.L. Chen, J.-H. Chu, J. G. Analytis, Z. K. Liu, K. Igarashi, H.-H. Kuo, X. L. Qi, S. K. Mo, R. G. Moore, D. H. Lu, M. Hashimoto, T. Sasagawa, S. C. Zhang, I. R. Fisher, Z. Hussain, and Z.X. Shen, Science 329, 659 (2010).

[2] M. Franz, Science 329, 639 (2010).

[3] L. A. Wray, S.-Y. Xu, Y. Xia, D. Hsieh, A. V. Fedorov, Y. S. Hor, R. J. Cava, A. Bansil, H. Lin, and M. Z. Hasan, Nature Phys. 7, 32 (2010).

[4] Q. Liu, C.-X. Liu, C. Xu, X.-L. Qi, and S.-C. Zhang, Phys. Rev. Lett. 102, 156603 (2009).

[5] J. E. Hirsch, Phys. Rev. Lett. 83, 1834 (1999).

[6] J. Sinova, D. Culcer, Q. Niu, N. A. Sinitsyn, T. Jungwirth, and A. H. MacDonald, Phys. Rev. Lett. 92, 126603 (2004).

[7] T. Kimura, Y. Otani, T. Sato, S. Takahashi, and S. Maekawa, Phys. Rev. Lett. 98, 156601 (2007).

[8] C. R. Ast, J. Henk, A. Ernst, L. Moreschini, M. C. Falub, D. Pacile, P. Bruno, K. Kern, and M. Grioni, Phys. Rev. Lett. 98, 186807 (2007).

[9] G. Bihlmayer, S. Blügel, and E. V. Chulkov, Phys. Rev. B 75, 195414 (2007).

[10] C.L. Kane and E. J. Mele, Phys. Rev. Lett. 95, 146802 (2005).

[11] L. Fu, C. L. Kane, and E. J. Mele, Phys. Rev. Lett. 98, 106803 (2007).

[12] M. König, S. Wiedmann, C. Brüne, A. Roth, H. Buhmann, L. W. Molenkamp, X.-L. Qi, and S.-C. Zhang, Science 318, 766 (2007).

[13] R. Yu, W. Zhang, H.-J. Zhang, S.-C. Zhang, X. Dai, and Z. Fang, Science 329, 61 (2010).

[14] X.-L. Qi, R. Li, J. Zang, and S.-C. Zhang, Science 323, 1184 (2009).

[15] D. Hsieh, Y. Xia, L. Wray, D. Quian, A. Pal, J. H. Dil, J. Osterwalder, F. Meier, G. Bihlmayer, C. L. Kane, Y.S. Hor, R. J. Cava, and M. Z. Hasan, Science 323, 919 (2009).

[16] L. Fu, Phys. Rev. Lett. 103, 266801 (2009).

[17] D. Hsieh, Y. Xia, D. Qian, L. Wray, F. Meier, J. H. Dil, J. Osterwalder, L. Patthey, A. V. Fedorov, H. Lin, A. Bansil,
D. Grauer, Y. S. Hor, R. J. Cava, and M.Z. Hasan, Phys. Rev. Lett. 103, 146401 (2009).

[18] Y. S. Hor, P. Roushan, H. Beidenkopf, J. Seo, D. Qu, J. G. Checkelsky, L. A. Wray, D. Hsieh, Y. Xia, S.-Y. Xu, D. Qian, M.Z. Hasan, N. P. Ong, A. Yazdani, and R. J. Cava, Phys. Rev. B 81, 195203 (2010).

[19] J. P. Perdew and Y. Wang, Phys. Rev. B 45, 13244 (1992).

[20] A. Gonis, Green Functions for Ordered and Disordered Systems, Studies in Mathematical Physics (North-Holland, Amsterdam, 1992), Vol. 4

[21] J. Henk, in Handbook of Thin Film Materials, edited by H. S. Nalwa (Academic Press, San Diego, 2002) Vol. 2, Chap. 10, p. 479.

[22] J. Zabloudil, R. Hammerling, L. Szunyogh, and P. Weinberger, Electron Scattering in Solid Matter (Springer, Berlin, 2005).

[23] P. J. Durham, B. L. Gyory, and A. J. Pindor, J. Phys. F 10, 661 (1980).

[24] C. Niu, Y. Dai, M. Guo, W. Wei, Y. Ma, and B. Huang, Appl. Phys. Lett. 98, 252502 (2011).

[25] H. Zhang, C.-X. Liu, X.-L. Qi, Z. Fang, and S.-C. Zhang, Nature Phys. 5, 438 (2009).

[26] Y.A. Bychkov and E.I. Rashba, J. Phys. C 17, 6039 (1984).

[27] R. Winkler, Spin-Orbit Coupling Effects in TwoDimensional Electron and Hole Systems (Springer, Berlin, 2003).

[28] S.-Y. Xu, Y. Xia, L. A. Wary, S. Jia, F. Meier, J. H. Dil, J. Osterwalder, B. Slomski, A. Bansil, H. Lin, R. J. Cava, and M. Z. Hasan, Science 332, 560 (2011).

[29] S. Basak, H. Lin, L. A. Wray, S.-Y. Xu, L. Fu, M.Z. Hasan, and A. Bansil, Phys. Rev. B 84, 121401 (2011).

[30] H.-J. Noh, H. Koh, S.-J. Oh, J.-H. Park, H.-D. Kim, J. D. Rameau, T. Valla, T. E. Kidd, P. D. Johnson, Y. Hu, and Q. Li, Europhys. Lett. 81, 57006 (2008).

[31] W. Jung, Y. Kim, B. Kim, Y. Koh, C. Kim, M. Matsunami, S.-I. Kimura, M. Arita, K. Shimada, J. H. Han, J. Kim, B. Cho, and C. Kim, Phys. Rev. B 84, 245435 (2011).

[32] T. Valla, Z.-H. Pan, D. Gardner, Y.S. Lee, and S. Chu, Phys. Rev. Lett. 108, 117601 (2012).

[33] Y. Okada, C. Dhital, W. Zhou, E. D. Huemiller, H. Lin, S. Basak, A. Bansil, Y.-B. Huang, H. Ding, Z. Wang, S. D. Wilson, and V. Madhavan, Phys. Rev. Lett. 106, 206805 (2011).

[34] T. M. Schmidt, R. H. Miwa, and A. Fazzio, Phys. Rev. B 84, 245418 (2011).

[35] O. V. Yazyev, J.E. Moore, and S. G. Louie, Phys. Rev. Lett. 105, 266806 (2010).

[36] S. Souma, K. Kosaka, T. Sato, M. Komatsu, A. Takayama, T. Takahashi, M. Kriener, K. Segawa, and Y. Ando, Phys. Rev. Lett. 106, 216803 (2011).

[37] S. V. Eremeev, Y. M. Koroteev, and E. V. Chulkov, JETP Lett. 91, 387 (2010).

[38] J. H. Dil, J. Phys. Condens. Matter 21, 403001 (2009).

[39] A. Kimura, E. E. Krasovskii, R. Nishimura, K. Miyamoto, T. Kadono, K. Kanomaru, E. V. Chulkov, G. Bihlmayer, K. Shimada, H. Namatame, and M. Taniguchi, Phys. Rev. Lett. 105, 076804 (2010).

[40] See Supplemental Material at http://link.aps.org/ supplemental/10.1103/PhysRevLett.108.206801 for hybridization of the Dirac state and bulk-electronic states. 